\title{
Research on Indoor Dynamic Positioning Algorithm Based on WiFi
}

\author{
Yan Zeng \\ Chengdu Vocational \& Technical College of Industry, Chengdu 610218, China
}

Keywords: wifi; weighted RSS algorithm; indoor positioning

\begin{abstract}
With the popularization of WiFi infrastructure in the indoor space and the rapid development of mobile intelligent devices, it brings new vitality to indoor positioning applications. The indoor positioning method based on WiFi is affected by the interference of indoor environment and the heterogeneity of mobile intelligent devices, thus resulting in great error in positioning accuracy. In this paper, the method of improving indoor positioning accuracy is proposed based on the indoor WiFi signal and the design of data collected by the multi-sensor set inside the mobile intelligent device. This paper proposes a weighted RSS indoor positioning algorithm; with the help ofsensor with high sensitivity builtin mobile device, it identifies the user behavior state. In the multi-floor indoor environment, it effectively improves the positioning accuracy of the algorithm; finally, we propose a WiFi indoor positioning algorithm based on sensor data fusion: hybrid multi-floor indoor positioning algorithm. The results showed that the multi-floor hybrid indoor positioning algorithm, based on the former one, organically integrated inertial positioning algorithm. As a result, it has a certain improvement in the positioning accuracy, positioning smoothness and real-time positioning.
\end{abstract}

\section{Introduction}

With the rapid development of WiFi infrastructure and the popularity of mobile intelligent devices, the positioning algorithm based on $\mathrm{WiFi}$ feature matching has become a promising indoor positioning algorithm. The mechanism of indoor positioning algorithm based onWiFi feature matching is to match by using real-time scanning WiFi signal data and the data in the sample database, and then to find out the position point of the most similar sample data as the location results of estimation.

Thepositioning accuracy of single indoor positioning algorithm based on WiFi feature matching is 1-5 meters.The indoor environment is dynamic and complex, which will affect the stability of WiFi signals, and then affect the positioning accuracy. Beyond that, there are a wide variety of mobile intelligent devices, there is a common problem of devices heterogeneity. The heterogeneity of the device can also significantly affect the matching result and reduce the positioning accuracy. This paper presents an indoor positioning algorithm based on WiFi feature matching of solving the indoor environment dynamic property, complexity and device heterogeneity: weighted RSS (Received Signal Strength WiFi) indoor positioning algorithm; in addition with the help of sensor with high sensitivitybuiltin mobile device, this paper identifies the user behavior, and in the multi-floor indoor environment, it effectively improves the positioning accuracy of the algorithm; finally, it puts forward a WiFi indoor positioning algorithm based on sensingdata fusion: hybrid multi-floor indoor positioning algorithm. 


\section{Methods}

The relationship between the WiFi signal intensity values of different wireless AP is comparatively stable. For example, WiFi signal strength value of AP2 received by different devices in the same place will be substantially greater than the WiFi signal strength values of AP1. The WiFi signal propagation model can be used to analyze this phenomenon, see(1):

$$
R S S(d)=R S S\left(d_{0}\right)+10 \lambda \log _{10} \frac{d}{d_{0}}+X
$$

In (1), $d$ is the distance between WiFi receiver and wireless AP, and $R S S(d)$ refers tothe signal attenuation of WiFi signal in the distance like $d . R S S\left(d_{0}\right)$ is a reference value, which is usually set to WiFi signal attenuation in the wireless distance AP1 meters. $\lambda$ is an impact factor, determined by the actual situation, and $\mathrm{X}$ is a Gauss variable. According to the propagation model, the WiFi signals received by different devices reflect the same relationship between them with the distance of wireless AP. It can be inferred that the relationship between the WiFi signals emitted by different APs is mainly affected by the distance, and this correlation is relatively stable, even if in the changing indoor environment and different mobile devices. As a result, it is possible to extract new WiFi signalfeatures by using the stable relative relation, and to design a robust indoor positioning algorithm based on WiFi feature matching: weighted RSS indoor positioning algorithm.

The weighted RSS indoor positioning algorithm is divided into two stages: off-line training data sample stage and online matching positioning stage.

The offline stage collects the WiFi signal data samples of indoor places, extracts the WiFi signal features, and constructs the WiFi feature fingerprint map of the whole indoor place. Assuming that there are $\mathrm{m}$ wireless APs, then the WiFi features of any place can be described as a vector, and the number of dimension in the vector is the number of wireless AP. That is: assuming that there are $\mathrm{n}$ positioning points $L=\left\{l_{1}, l_{2}, \ldots, l_{n}\right\}$ in the indoor place, and the vector $R_{j}=\left(r j_{1}, r j_{2}, \ldots, r j_{m}\right)$ in the position of $l_{j}$, in which $r j_{i}$ refers tothe wireless $A P_{j}$ WiFisignal strength value received by the site, then the WiFi feature weight of the positioning point $l_{j}$ is seen in (2):

$$
D_{j}=\left\{\left(r_{j}^{1}, s\left(r_{j}^{1}\right)\right),\left(r_{j}^{2}, s\left(r_{j}^{2}\right)\right), \ldots,\left(r_{j}^{m}, s\left(r_{j}^{m}\right)\right)\right\}
$$

In the formula, $s\left(r_{j}^{1}\right)$ is the index value of the vector in the $\mathrm{Rj}$ after being ordered. For example, in the actual data, there is $R=(-58,-39,-46)$, and then the weighted WiFi feature $D=(-58,3),(-39,1)$, $(-46,2)\}$.

After calculating the WiFi feature weight of the device at a certain point, we can match the WiFi feature in the fingerprint database. Assuming that $D_{t r}$ is a WiFi feature in the fingerprint database, and $D_{t e}$ is the real-time feature of the device, then the weighting factor $W_{t r, t e}$ is shown in(3):

$$
W_{t r, t e}=\left\{W_{1}, W_{2}, \ldots, W_{m}\right\}
$$

$W_{i}$ is shown in (4):

$$
W_{i}=1-\frac{\left|s\left(R_{t r}^{i}\right)-s\left(R_{t e}^{i}\right)\right|}{\max \left\{s\left(R_{t r}^{i}\right), s\left(R_{t e}^{i}\right)\right\}}, \quad 1 \leq i \leq m
$$

Then, we can calculate the Euclidean distance of $D_{t r}$ and $D_{t e}$, as shown in (5):

$$
\operatorname{Dist}\left(D_{t r}, D_{t e}\right)=\sqrt{\sum_{i=1}^{m} w_{i}\left(r_{t r}^{i}-r_{t e}^{i}\right)^{2}}
$$

After obtaining this distance, the nearest neighbor algorithm can be used to estimate the positioning point. 
The indoor positioning algorithm framework diagram is shown in Figure 1. It is mainly composed of four modules: (1) weighted RSS algorithm module; (2) inertial positioning module; (3) floor recognition module; and (4)walking state recognition module. The algorithm can be divided into two stages: off-line training stage and on-line positioning stage.
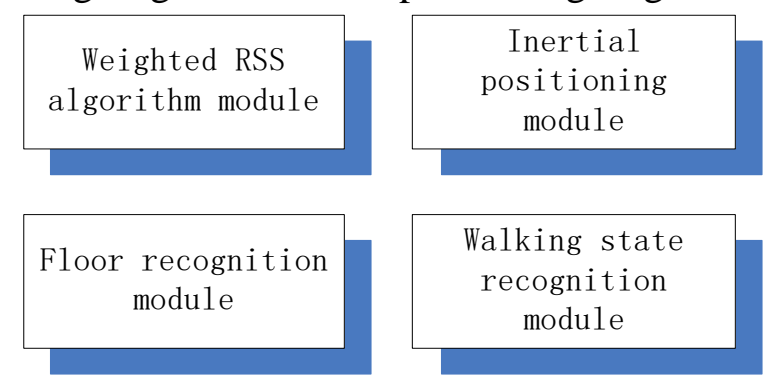

Figure 1 Hybrid multi-floor indoor positioning algorithm module

In the off-line training stage, the WiFi data of the whole indoor floor is first of all collected, and each sample data includes: WiFi signal strength, wireless AP SSID, MAC address and acquisition point number of wireless AP. After the completion of the data acquisition, we train two features sample database. The first is the WiFi feature sample database needed by the weighted RSS algorithm, and the second is FLD training samples required by the floor recognition algorithm. At the same time, we acquire the accelerometer sampling data of the user's behavioral states, used for the recognition and inertial positioning of the user's behavior state.

In the online positioning stage, first of all, through the walking state recognition algorithm, we determine whether the floor where the user is located changes. If it sends change, it will trigger the floor recognition algorithm, and the floor recognition algorithm will identify the number of floors of the users. Then, through the inertial positioning, we can estimate the range of motion and the approximate direction of the user in the plane of adjacent several positioning. Next, we take the number of floors and inertial positioning results as the basis, to determine the range of the user's position in the certain floor. In addition, we select the corresponding child sample database from the WiFi feature sample database as the sample data for the positioning matching. At last, we make use of weighted RSS algorithm to obtain the positioning results.

\section{Results}

The performance of the proposed algorithm is evaluated. The site of the performance evaluation is the experimental building of the author. The building has 6 floors, and we selected the first floor to the fifth floor of the building. According to the building layout of the different floors, we divided the first floor into 169 squares, and divided the second floor, the third floor, the fourth floor and the fifth floor into 139 squares. The distance between the adjacent squares is about 1 meter.According to the squares divided, we acquired the WiFi signal sample data, and constructed the WiFi feature database. The mobile intelligent devices used in the experiment are three smart phones and a tablet computer. In order to investigate the effects of heterogeneous devices, a test method that we can take is: Nexus 7 and HUAWEI Ascend P6 used for the acquisition of WiFi signal samples. Later, we respectively use the four devices for algorithm performance evaluation. In this way, it tested the positioning performance in the two cases of non-deviceheterogeneity and device heterogeneity.

We compared multi-floor hybrid indoor positioning algorithm and weighted RSS positioning algorithm. The test site is the same as theprevious. Figure 2 is the positioning error distribution of the two algorithms. We can see that the positioning accuracy of the hybrid positioning algorithm is higher than that of the weighted RSS algorithm with floor recognition. Figure 3 is thetime-consuming calculation of the above two algorithms. The time-consuming calculation of 
hybrid positioning algorithm and weighted RSS algorithm with floor recognition takes significantly less than the weighted RSS algorithm without floor recognition. At the same time, because of the addition of the inertial positioning, it can further reduce the space complexity of positioning algorithm, which further reduces the algorithm computation time-consuming. Compared with the weighted RSS indoor positioning algorithm with floor recognition, multi-floor hybrid indoor positioning algorithm proposed in this paper, on the basis of the former one, organically fuses inertial positioning algorithm. As a result, the positioning accuracy, positioning smoothness and real-time positioning were improved to some extent.
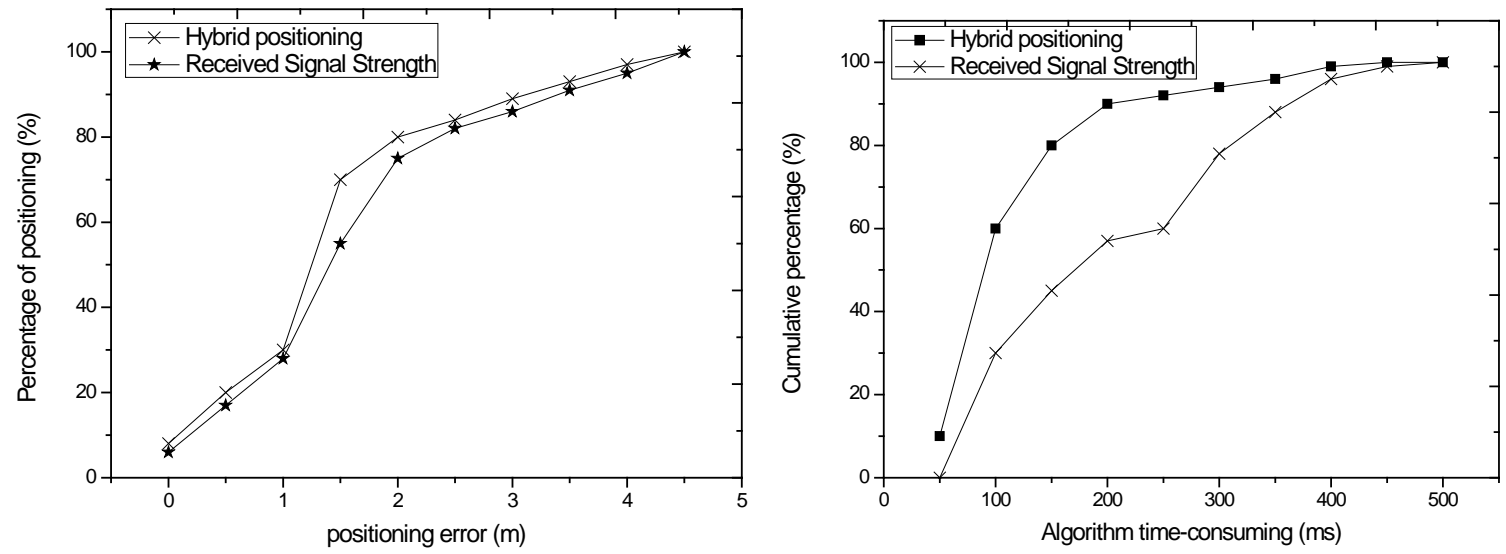

Figure 2Positioning error analysisFigure 3Positioning algorithm time-consuming

\section{Conclusion}

According to the complex and changeable indoor environment and outbreak developing mobile intelligent devices, we put forward a kind of WiFi indoor positioning algorithm based on sensor data fusion. This algorithm solves the unstable WiFi signal in the multi-floor indoor environment and mobile heterogeneous problems. At the same time, the algorithm adds with inertial positioning and floor recognition for assisting the positioning, which improves the positioning accuracy. In addition, to a certain extent, it overcomes the limitation of single indoor positioning algorithm based on WiFi feature matching, and effectively reduces the space complexity of positioning algorithm, so the algorithm has better real-time property and practicability. The algorithm has good robustness and scalability in the face of complex and changeable dynamic indoor environment and the ever-changing mobile intelligent devices. Finally, we evaluate the performance of the weighted RSS algorithm and hybrid multi-floor indoor positioning algorithm, andthe results are satisfactory.

\section{References}

[1].Mo, Y., Zhang, Z., Lu, Y. and Agha, G., 2015. A Novel Technique for Human Traffic based Radio Map Updating in Wi-Fi Indoor Positioning Systems. TIIS,9(5), pp.1881-1903.

[2].Li, L., Shen, G., Zhao, C., Moscibroda, T., Lin, J.H. and Zhao, F., 2014, September. Experiencing and handling the diversity in data density and environmental locality in an indoor positioning service. In Proceedings of the 20th annual international conference on Mobile computing and networking(pp. 459-470). ACM.

[3].Faragher, R. and Harle, R., 2014, September. An analysis of the accuracy of bluetooth low energy for indoor positioning applications. In Proceedings of the 27th International Technical Meeting of The Satellite Division of the Institute of Navigation (ION GNSS+ 2014), Tampa, FL, USA (Vol. 812, p. 2). 
[4].Howell, C., 2016. Applications of Bluetooth Radio Technology and Indoor Positioning in the Hospital.

[5].Nilsson, J.O., Rantakokko, J., Handel, P., Skog, I., Ohlsson, M. and Hari, K.V.S., 2014, May. Accurate indoor positioning of firefighters using dual foot-mounted inertial sensors and inter-agent ranging. In Position, Location and Navigation Symposium-PLANS 2014, 2014 IEEE/ION (pp. 631-636). IEEE.

[6].Xu, H., Ding, Y., Wang, R., Shen, W., \& Li, P. (2016). A novel Radio Frequency Identification three-dimensional indoor positioning system based on trilateral positioning algorithm. Journal of Algorithms \& Computational Technology, 10(3), 158-168.

[7].Zhang, W., Hua, X., Yu, K., Qiu, W., \& Zhang, S. (2016, October). Domain clustering based WiFi indoor positioning algorithm. In Indoor Positioning and Indoor Navigation (IPIN), 2016 International Conference on (pp. 1-5). IEEE.

[8].Wang, H., Zhao, Z., Hu, J., Qu, Z., \& Feng, H. (2016, May). Study on improvement of fingerprint matching algorithm in wireless LAN based indoor positioning system. In Software Engineering, Artificial Intelligence, Networking and Parallel/Distributed Computing (SNPD), 2016 17th IEEE/ACIS International Conference on (pp. 275-280). IEEE.

[9].Gu, W., Kavehrad, M., \& Aminikashani, M. (2016, February). Three-dimensional indoor light positioning algorithm based on nonlinear estimation. In SPIE OPTO (pp. 97720V-97720V). International Society for Optics and Photonics.

[10].Guan, W., Wu, Y., Wen, S., Chen, H., Yang, C., Chen, Y., \& Zhang, Z. (2017). A novel three-dimensional indoor positioning algorithm design based on visible light communication. Optics Communications, 392, 282-293. 